\title{
Effect of sodium-glucose transport protein 2 inhibitors on serum uric acid: an outpatient based prospective interventional study
}

\author{
Dipan Saha, Subhayan Das*, Ramnarayan Maiti, Swarnali Paul
}

Department of Pharmacology, Midnapore Medical College, Midnapore, West Bengal, India

\author{
Received: 01 April 2020 \\ Revised: 11 May 2020 \\ Accepted: 12 May 2020 \\ *Correspondence: \\ Dr. Subhayan Das, \\ Email: drsubhayandas198@gmail.com
}

Copyright: $@$ the author(s), publisher and licensee Medip Academy. This is an open-access article distributed under the terms of the Creative Commons Attribution Non-Commercial License, which permits unrestricted non-commercial use, distribution, and reproduction in any medium, provided the original work is properly cited.

\begin{abstract}
Background: Hyperuricemia is considered to be associated with increased risk of cardiovascular diseases. It has been found to be related to features of metabolic syndromes like hyperglycemia and dyslipidemia. Sodium-glucose transport protein 2 (SGLT2) inhibitors are known to have a decremental effect on serum uric acid level. We evaluated the effect of SGLT2 inhibitors on serum uric acid of diabetic population.

Methods: In this prospective study we recruited 50 type 2 diabetes mellitus (T2DM) patients who were on metformin monotherapy and were having inadequate glycemic control. Patients were prescribed SGLT2 inhibitors as an add-on therapy. Serum uric acid of the subjects was measured at the baseline and after 3 months. The primary outcome was to observe changes in serum uric acid (SUA) levels from the baseline to the end of the study. Glycemic changes were determined by observing the changes in glycated haemoglobin (HbA1c) levels (if any).

Results: The study population was predominantly male (82\%). BMI wise most of the subjects (44\%) were overweight. Mean HbA1c of total population was $7.65 \pm 0.5$, whereas mean serum uric acid was $6.31 \pm 0.72$ at the baseline. After 3 months $\mathrm{HbA1C}$ and serum uric acid levels were $7.45 \pm 0.50$ and $6.06 \pm 0.64$ respectively. Both the changes were statistically significant $(\mathrm{p}<0.005)$.

Conclusions: SGLT2 inhibitors could improve glycemic control and lower SUA levels in patients with uncontrolled T2DM. Randomized study with bigger sample size and longer study period are required to further demonstrate the effect of SGLT2 inhibitors on serum uric acid and explore the potential underlying mechanisms.
\end{abstract}

Keywords: SGLT, Uric acid, Diabetes

\section{INTRODUCTION}

Type 2 diabetes mellitus (T2DM) is one of the most common endocrine disorders encountered in clinical practice globally. In the US prevalence of DM was found to be around $14 \%$ whereas in case of pre-diabetes the number was $38 \%$ in the year 2011-12. ${ }^{1}$ Type 2 diabetes is progressive in nature and has an underlying pathophysiology which is multifactorial and quite complex in nature. The disease often requires different class of pharmacological agents in individual patients to achieve optimum glycemic control. ${ }^{2}$ many new therapeutic approaches evolving for last few years in the treatment of DM. Of these therapies, the newest types are incretin-based therapies and the sodium-glucose cotransporter-2 (SGLT-2) inhibitors.

Many studies in the past have indicated that there is a close association between T2DM and cardiovascular diseases (CVD). Hypertension, dyslipidemia, obesity, endothelial dysfunctions are the features we can commonly relate to the state of persistent hyperglycemia. ${ }^{3}$ Hyperuricemia is an increasingly 
common medical problem in both developed as well as developing countries. It is suggested that this condition is closely related to the other features of metabolic syndrome like dyslipidemia and hypertension. ${ }^{4}$ Moreover recent studies have indicated that high level of serum uric acid level may give rise to CVD like atherosclerotic heart diseases and atrial fibrillation. The relation between hyperuricemia and coronary artery disease is established worldwide, although the relationship between the two is a complex one. High levels of uric acid can give rise to CVD by promoting various pathophysiological mechanisms like forming uric acid crystals, inducing NO production, exerting oxidative stress, promoting inflammatory reactions and others. ${ }^{5}$ SGLT2 inhibitors lower blood glucose in an insulin-independent manner by reducing the reabsorption of glucose from the renal filtrate. In addition to lowering the blood glucose level it helps in weight reduction. Uric acid levels are generally higher in diabetic population. Several clinical trials and meta-analysis have clearly demonstrated that usage of SGLT2 inhibitors in diabetic individuals can lower the circulating uric acid levels consistently. Greater reduction in serum uric acid level was observed among the patients with high glycated haemoglobin (HbA1c) levels. ${ }^{6}$ It is evident that treating a diabetic individual with SGLT2 inhibitors as a monotherapy or add on can significantly increases uric acid excretion, reduces circulating uric acid and improves parameters of $\mathrm{CV}$ and renal function. This study aims at finding the impact of SGLT2 inhibitors on serum uric acid level when used as a add on therapy to metformin in diabetic individuals.

\section{METHODS}

It was a prospective interventional study involving patients with type 2 diabetes mellitus who were on metformin monotherapy in optimum dosage ( 2 gm daily) and having inadequate glycemic control. A total of 50 subject attending the Internal Medicine outpatient department of Midnapore medical college and hospital, aged between 30-60 years were recruited for the study. Total duration of the study was 12 weeks (November 2019 to February 2020). Institutional ethics committee's approval was obtained prior to initiating our study. Written informed consent was received from each participant. Blood samples were sent to measure biochemical parameters like serum uric acid, HbA1c at the baseline. Personal history taking and other clinical parameters were screened at the beginning of the study. All the subjects were assessed for their respective ASCVD risk for 10 years using the mobile application of American College of Cardiology. Those subjects receiving metformin $2 \mathrm{gm}$ daily but having ASCVD risk $>7.5 \%$ and $\mathrm{HbAlc}>7 \%$ were included in the study and put on SGLT 2 inhibitor class of drugs as an add-on to metformin therapy. Subjects having eGFR <45 (calculated using MDRD formula) and history of recent genitourinary infection were excluded. Other clinical and non-clinical parameters were thoroughly screened to assess the subjects' compatibility with this class of anti- diabetic agents. With the help of endocrinologist adequate dosage regimens were finalized for each participant. Canagliflozin and dapagliflozin were the drugs used in our study. After 12 weeks serum uric acid level and HbA1c were measured and compared with the baseline values. All the subjects were put on strict lowcalorie diet which is suitable to their respective glycemic status. Special emphasis was given for life style modification for throughout the study period as well. Foods or drugs which can interfere with the serum uric acid level were advised to be excluded from the diet of the participants throughout the study period.

\section{Statistical analysis}

Data was entered on Microsoft excel. Continuous variables were summarized as mean and standard deviation. Statistical analysis was performed using SPSS version 21. Paired $t$ and unpaired test were used for intragroup and intergroup comparison of the values respectively. A p-value $<0.05$ was considered significant. All the analysis was carried out at the Department of Pharmacology, Midnapore Medical College, West Bengal.

\section{RESULTS}

The study was conducted to find out the impact of SGLT2 inhibitors on serum uric acid level when used as an add on therapy to metformin in diabetic individuals. Among the pts, aged 35-70 years, attending general medicine outpatient Department of Midnapore Medical College. 50 patients met the inclusion criteria and got enrolled in our study. The study subjects were predominantly male $(82 \%)$ as shown in (Figure 1$)$, with the mean age of 56.16 years respectively. BMI wise most of the subjects $(44 \%)$ belonged to overweight category followed by obese category $(32 \%)$ and normal category (24\%) (Figure 2).

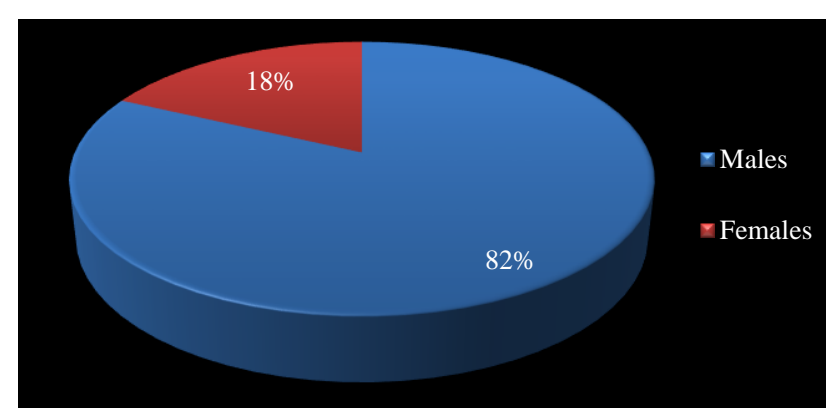

Figure 1: Gender wise distribution of study population.

26 out of 50 patients were on tab canaglifozin $200 \mathrm{mg}$ $(52 \%)$ and rest $24(48 \%)$ were on tab dapaglifozin $10 \mathrm{mg}$ as an add on therapy to tab metformin. In the canagliflozin group the mean HbA1c was 7.6192 \pm 0.510 and the mean serum uric acid (SUA) level was $6.2346 \pm 0.677$ at the baseline. On the other hand, the 
mean HbA1c and the mean SUA of the dapaglifozin group were $7.6958 \pm 0.499$ and $6.391 \pm 0.771$ respectively at the baseline. At the end of 3 months, the mean HbA1c of the dapaglifozin group was 7.512 \pm 0.467 and the mean HbA1C of the canaglifozin group was $7.400 \pm 0.538$, whereas the mean serum uric acid level of the dapaglifozin group was $6.095 \pm 0.675$ and in canaglifozin group it was found to be $6.038 \pm 0.624$ (Figure 3). Both the changes were found to be statistically insignificant
(Table1). However, when the total study population is considered, mean HbA1c was $7.65 \pm 0.5$, whereas mean serum uric acid was $6.31 \pm 0.72$ at the baseline. After 3 months $\mathrm{HbA} 1 \mathrm{C}$ and serum uric acid levels of total population were found to be $7.45 \pm 0.50$ and $6.06 \pm 0.64$ respectively. Both the changes were statistically significant $(\mathrm{p}<0.005)$ (Figure 4 and Table 1$)$. No adverse drug reactions were reported throughout the study period.

Table 1: Comparison between mean HbA1c and mean SUA levels at baseline and after 3 months in total population and individual groups of patients receiving dapagliflozin and canagliflozin.

\begin{tabular}{|llll|}
\hline S. no. & Baseline level (mean \pm SD) & After 3 months $($ mean \pm SD) & P value \\
\hline Mean HbA1c (total population) & $7.65 \pm 0.501$ & $7.45 \pm 0.503$ & $\geq 0.05$ \\
\hline Mean SUA (total population) & $6.310 \pm 0.720$ & $6.066 \pm 0.643$ & $\geq 0.05$ \\
\hline Mean HbA1c (CANA) & $7.619 \pm 0.510$ & $7.400 \pm 0.538$ & $\leq 0.05$ \\
\hline Mean SUA (CANA) & $6.234 \pm 0.677$ & $6.038 \pm 0.624$ & $\leq 0.05$ \\
\hline Mean HbA1c (DAPA) & $7.695 \pm 0.499$ & $7.512 \pm 0.467$ & $\leq 0.05$ \\
\hline Mean SUA (DAPA) & $6.391 \pm 0.771$ & $6.095 \pm 0.675$ & $\leq 0.05$ \\
\hline
\end{tabular}

Values of serum uric acid (SUA) are expressed in $\mathrm{mg} / \mathrm{dl}$, values of HbA1c are in \%.

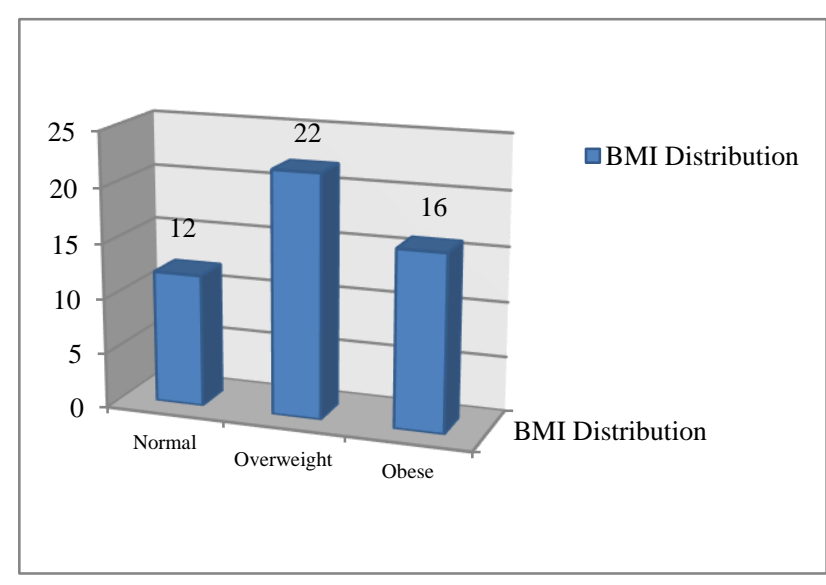

Figure 2: BMI category distribution of study population (WHO Asia-Pacific classification).

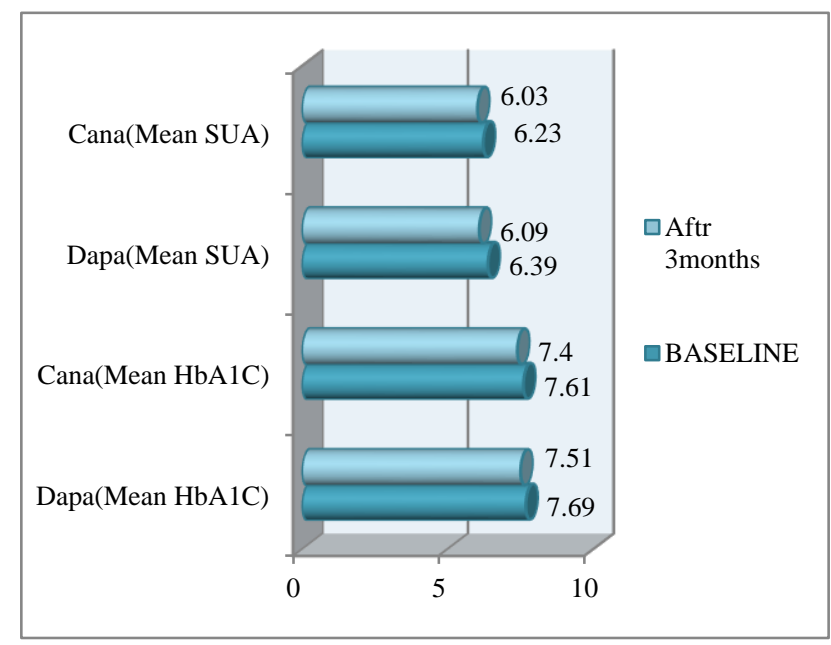

Figure 3: Intra-group comparison between baseline mean HbA1c and mean SUA values and values obtained after 12 weeks in both dapagliflozin and canagliflozin group.

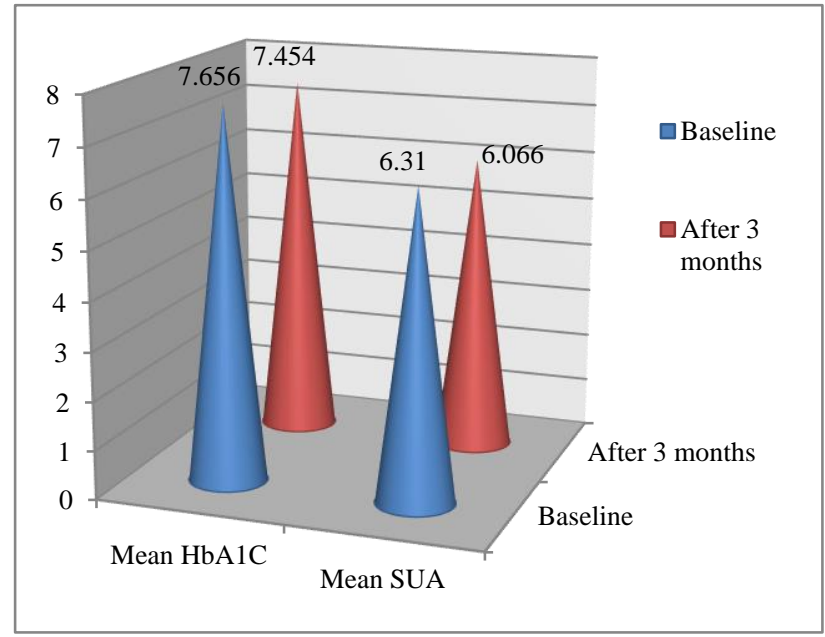

Figure 4: Comparison between mean HbA1c levels and mean SUA levels of the total study population at baseline and after 3 months.

\section{DISCUSSION}

The present study elucidated the impact of the SUA lowering effect that is commonly observed in response to SGLT2 inhibitors in diabetic population which can be an additional ploetrophic benefit in addition to glycemic control. When glycemic control is concerned, total population achieved significant reduction in $\mathrm{HbA} 1 \mathrm{c}$ values after 3 months when compared to baseline, various randomized studies, meta-analysis have shown similar efficacy of SGLT2 inhibitors in the past. ${ }^{7}$ This group of oral anti diabetic drugs acts in insulin independent manner, and promotes glycosuria by inhibiting glucose reabsorption from the proximal convoluted tubule of nephron. Studies have demonstrated dose dependent increment in glycosuria and improvement in glycemic parameter like oral glucose tolerance test in the past. $^{8}$ 
Hyperuricemia is common biochemical finding in patients with T2DM. significant reduction in serum uric acid level have been observed when diabetic patients are treated with drugs like canagliflozin. ${ }^{9}$ Similar results were observed in a randomized control study conducted in china, which showed dapagliflozin effectively reduced serum uric acid level of hospitalized patients having inadequate glycemic control. ${ }^{10}$ Both these results are consistent with finding of our study, as significant reduction in uric acid as well as $\mathrm{HbAlc}$ were observed when total population is concerned includes both dapagliflozin and canagliflozin groups. It can be said SGLT2 inhibitors as a whole has a decremental effect on serum uric acid, apart from the drugs used in our study, another molecule in this class i.e., empagliflozin also showed similar effect on serum uric acid as well as blood pressure of diabetic population. ${ }^{11}$ It is widely suggested that metformin is the drug that every drug naïve diabetic patients should be started with unless contraindicated. ${ }^{12}$ When choosing the add on therapy to metformin factors like smoking, co-morbid conditions like heart failure, family history of CVD should be taken into account by every clinician. Elevated serum uric acid is associated with conditions mentioned above and should be kept below the optimum level in diabetic patients. Like the findings of our study several meta-analysis and RCTs have demonstrated the fact that SGLT2 inhibitors can provide us the pleotrophic benefit in form of reduction in serum uric acid levels when used in appropriate patients in adequate dosage regimens. ${ }^{13}$ Although we were not able to strictly monitor factors like diet, lifestyle, usage of other medications by individual subjects throughout the study period.

\section{CONCLUSION}

In conclusion, SGLT2 inhibitors like canagliflozin and dapagliflozin provides the evidence that they reduce uric acid and HbAlc. These findings suggest the beneficial effects of SGLT2 inhibitors on the cardiovascular risk factors in patients with T2 DM.

Funding: No funding sources Conflict of interest: None declared

Ethical approval: The study was approved by the Institutional Ethics Committee

\section{REFERENCES}

1. Hsia DS, Grove O, Cefalu WT. An Update on Sodium-Glucose Co-transporter-2 Inhibitors for the Treatment of Diabetes Mellitus. Curr Opin Endocrinol Diabetes Obes. 2017;24:73-9.

2. Monami M, Nardini C, Mannucci E. Efficacy and safety of sodium glucose co-transport-2 inhibitors in type 2 diabetes: a meta-analysis of randomized clinical trials. Diabetes, Obesity and Metabolism. 2014;16:457-66.

3. Desouza CV, Gupta N, Patel A. Cardiometabolic Effects of a New Class of Antidiabetic Agents. Clinical Therapeutics. 2015;1:1-17.

4. Liying C, Wenhua Z, Zhouwen C, Honglei D, Jing R, Jianhua $C$, et al. Relationship between hyperuricemia and metabolic syndrome. J Zhejiang Univ Sci B. 2007;8(8):593-8.

5. Wang H, Zhang H, Sun L, Guo W. Am J Transl Res. 2018;10(9):2749-63.

6. Bailey CJ. Uric acid and the cardio-renal effects of SGLT2 inhibitors. Diabetes Obes Metab. 2019;21:1291-8.

7. Bailey CJ, Gross JL, Pieters A, Bastien A, List JF. Effect of dapagliflozin in patients with type 2 diabetes who have inadequate glycaemic control with metformin: a randomised, double-blind, placebo controlled trial. Lancet. 2010;375:2223-33.

8. Komoroski B, Vachharajani N, Feng Y, Li L, Kornhauser D, Pfister M. Dapagliflozin, a Novel, Selective SGLT2 Inhibitor, Improved Glycemic Control Over 2 Weeks in Patients with Type 2 Diabetes Mellitu. Clin Pharmacol Ther. 2009;85(5):513-9.

9. Davies MJ, Trujillo A, Vijapurkar U, Damaraju CV, Meininger G. Effect of canagliflozin on serum uric acid in patients with type 2 diabetes mellitus. Diabetes Obes Metab. 2015;17(4):426-9.

10. Hao Z, Xiao H, Shao H, Tian F. Effects of dapagliflozin on serum uric acid levels in hospitalized type 2 diabetic patients with inadequate glycemic control: a randomized controlled trial. Therapeutics Clin Risk Management. 2018;14:240713.

11. Zhao D, Liu H, Dong P. Empagliflozin reduces blood pressure and uric acid in patients with type 2 diabetes mellitus: a systematic review and meta-analysis. J Human Hypertension. 2018;33(4):327-39.

12. Rojas LBA, Gomes MB. Metformin: an old but still the best treatment for type 2 diabetes. Diabetology Metab Syndrome. 2013;5 (1):6.

13. Zhao Y, Xu L, Tian D, Xia P, Zheng H, Wang L, et al. Effects of sodium-glucose co-transporter 2 (SGLT2) inhibitors on serum uric acid level: a metaanalysis of randomized controlled trials. Diabetes Obes Metab. 2018;20(2):458-62.

Cite this article as: Saha D, Das S, Maiti R, Paul S. Effect of sodium-glucose transport protein 2 inhibitors on serum uric acid: an outpatient based prospective interventional study. Int J Basic Clin Pharmacol 2020;9:962-5. 